\title{
Reformulation of the Generalized Curvature by Using Decomposition Technique
}

\section{Tahir H. Ismail \\ Dept. of Mathematics \\ College of Computer Science \& Math \\ University of Mosul}

\section{Received}

30/08/2006
Ibrahim O. Hamad

Dept. Of Mathematics

College of Science

Salahaddin University-Hawler

Accepted

10/12/2006

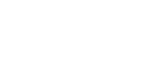

في هذا البمث. نحاطل إنشاء صيغة جدية لقوس معم م للمنحذ مي الم ستوي (t) معتمدين على ظرية التحل وذلك للحصول على نقوس معمم للمنحنيل جبري ـة مـ ستخدمن

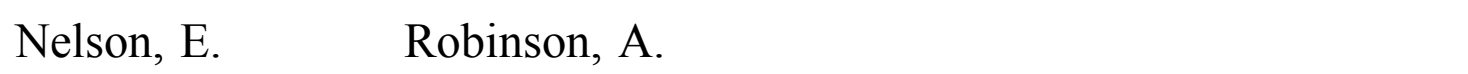
بلسلوب مظقي.

\begin{abstract}
The aim of this paper is to establish a new formula of generalized curvature discussed with the help of decomposition theorem which is used to obtain generalized curvature for algebraic curves by using some concepts of nonstandard analysis given by Robinson A. and axiomatized by Nelson E.
\end{abstract}

\section{1-Introduction: -}

Among the difference expressions of the generalized curvature, there is one which can be expressed by using only one point infinitely close to a singular point of a standard curve and this will be our consideration in this paper. This is derived from generalized curvature form [10], with the help of decomposition theorem.

The following definitions of nonstandard analysis will be needed throughout this paper. [4], [5], [9], [11], [12], [15], [16], [17]

A real number $\boldsymbol{x}$ is called unlimited if and only if $|\boldsymbol{x}|>\boldsymbol{r}$ for all positive standard real numbers, otherwise it is called limited.

The set of all unlimited real numbers denoted by $\overline{\mathbf{R}}$, and the set of all limited real numbers denoted by $\underline{\mathbf{R}}$

A real number $\boldsymbol{x}$ is called infinitesimal if and only if $|\boldsymbol{x}|<\boldsymbol{r}$ for all positive standard real numbers $\boldsymbol{r}$.

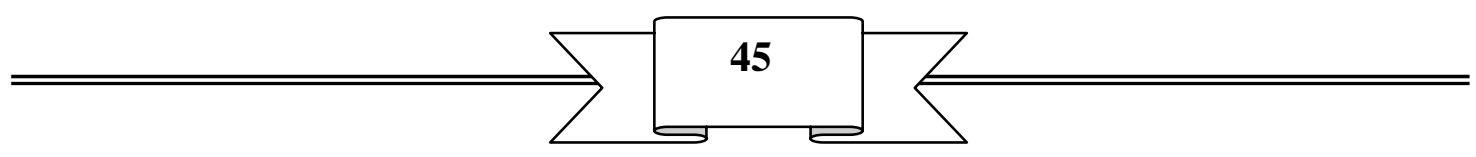


Two real numbers $x$ and $y$ are said to be infinitely close if and only if $\boldsymbol{x}-\boldsymbol{y}$ is infinitesimal and denoted by $\boldsymbol{x} \cong \boldsymbol{y}$.

If $\boldsymbol{x}$ is a limited number in $\mathbf{R}$, then it is infinitely close to a unique standard real number, this unique number is called the standard part of $\boldsymbol{x}$ or shadow of $\boldsymbol{x}$ denoted by $\operatorname{st}(x)$ or ${ }^{0} \boldsymbol{x}$.

Every set or element defined in a classical mathematics is called standard.

Any set or formula which does not involve new predicates "standard, infinitesimals, limited,...etc" is called internal, otherwise is called external.

Let $\quad \alpha: \mathbf{I} \rightarrow \mathbf{R}^{3}, \mathbf{I}=(a, b)$, be a curve parametrized by an arc length $s$, and its tangent vector be $\alpha$ ' which has a unit length, then the measure of the ratio of change of the angle with neighboring tangents made with the tangent at $\mathrm{s}$ is known as a curvature of the curve $\alpha$ at $s$ ,and it is given by $\left|\boldsymbol{g}^{\prime \prime}(\boldsymbol{s})\right|$ and denoted by $\boldsymbol{\kappa}(\boldsymbol{s})$

\section{Theorem 1.1 [10]}

Let $A=\gamma(\boldsymbol{t})$ be a standard point on the planar curve $\gamma$, and let $B$ and $C$ be two points infinitely close to the point $A$, then

\section{Theorem 1.2 [10]}

$$
\frac{\tan \hat{A}}{\|\overrightarrow{B C}\|}=\frac{\overrightarrow{A C} \times \overrightarrow{A B}}{\|\overrightarrow{B C}\|^{3}} \cong \frac{\overrightarrow{B C} \times \overrightarrow{A B}}{\|\overrightarrow{B C}\|^{3}}
$$

Let $A$ be a standard point on the curve $\gamma$, and let $B$ and $C$ be two points infinitely close to the point $A$, then the generalized curvature $\kappa_{G}$ of the curve $\gamma$ at the point $A$ is given by: $\kappa_{G} \cong \frac{|\tan \hat{A}|}{\|\overrightarrow{B C}\|} \cong \frac{|\overrightarrow{A C} \times \overrightarrow{A B}|}{\|\overrightarrow{B C}\|^{3}}$.

2- Decomposition Technique for Calculating the Generalized Curvature

According to the applications of decomposition theorem to algebraic equations, we give an expression of the generalized curvature, which does not involve the parametric but a point which infinitely close to the considered standard part.

\section{Theorem 2.1(Decomposition Technique Theorem)}

If $\boldsymbol{M}$ is a limited point of $\mathbf{R}^{\mathbf{2}}$ whose shadow is ${ }^{o} \boldsymbol{M}=\boldsymbol{M}_{o}$, then for $\boldsymbol{M} \neq \boldsymbol{M}_{\boldsymbol{o}}$ there exist two standard vectors ${\overrightarrow{V_{1}}}_{1}$ and $\overrightarrow{\boldsymbol{V}_{2}}$ linearly independent 
and two real infinitesimal numbers $\varepsilon_{1}$ and $\varepsilon_{2}$ such that $\vec{M} \cong \vec{M}_{o}+\varepsilon_{1} \vec{V}_{1}+\varepsilon_{1} \varepsilon_{2} \vec{V}_{2}$.

Proof:

Since ${ }^{o} \boldsymbol{M}=\boldsymbol{M}_{o}$ then there exist an infinitesimal $\vec{\eta}$ such that

$$
\overrightarrow{\boldsymbol{M}}=\overrightarrow{\boldsymbol{M}}_{o}+\vec{\eta}
$$

where $\boldsymbol{M}, \boldsymbol{M}_{o}, \eta \in \mathbf{R}^{2}$.

Put $\varepsilon_{1}=\left\|\overrightarrow{\boldsymbol{M}}-\overrightarrow{\boldsymbol{M}}_{o}\right\|$ and $\quad \overrightarrow{\boldsymbol{P}}_{1}=\frac{\overrightarrow{\boldsymbol{M}}-\overrightarrow{\boldsymbol{M}}_{o}}{\varepsilon_{1}}$

then $\overrightarrow{\boldsymbol{P}}_{1}$ is a unit direction vector.

Also put $\overrightarrow{\boldsymbol{V}_{1}}=\stackrel{o}{\boldsymbol{P}_{1}}$ then there exist an infinitesimal $\vec{\zeta}$ such that

$$
\vec{P}_{1}=\vec{V}_{1}+\vec{\zeta}
$$

Put $\quad \varepsilon_{2}=\left\|\overrightarrow{\boldsymbol{P}}_{\mathbf{1}}-\overrightarrow{\boldsymbol{V}}_{1}\right\|, \quad \overrightarrow{\boldsymbol{P}_{2}}=\frac{\overrightarrow{\boldsymbol{P}_{1}}-\overrightarrow{\boldsymbol{V}}_{1}}{\varepsilon_{2}}$, and $\quad \overrightarrow{\boldsymbol{V}_{2}}={ }^{o} \overrightarrow{\boldsymbol{P}_{2}}$, then there exist an infinitesimal $\xi$ such that $\overrightarrow{\boldsymbol{P}_{2}}=\overrightarrow{\boldsymbol{V}}_{2}+\vec{\xi}$

Combine equations (2.1.2)-(2.1.4) and substitute them in equation (2.1.1) we get that $\overrightarrow{\boldsymbol{M}}=\overrightarrow{\boldsymbol{M}_{o}}+\varepsilon_{1} \overrightarrow{V_{1}}+\varepsilon_{1} \varepsilon_{2} \overrightarrow{V_{2}}+\varepsilon_{1} \varepsilon_{2} \vec{\xi}$ Thus

$$
\vec{M} \cong \vec{M}_{o}+\varepsilon_{1} \vec{V}_{1}+\varepsilon_{1} \varepsilon_{2} \vec{V}_{2} .
$$

\section{Corollary 2.2}

If $\boldsymbol{M}$ is limited point of $\mathbf{R}^{\mathbf{2}}$ whose shadow is ${ }^{o} \boldsymbol{M}=\boldsymbol{M}_{o}$, then for ${ }^{o} \boldsymbol{M}=\boldsymbol{M}_{o}$ there exist two real infinitesimal numbers $\varepsilon_{1}$ and $\varepsilon_{2}$ such

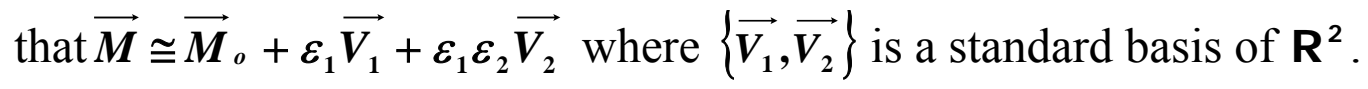

Proof:

Since ${ }^{{ }^{o} \boldsymbol{M}}=\overrightarrow{\boldsymbol{M}}_{\text {ot }}$ then there exist an infinitesimal $\vec{\eta}$ such that $\overrightarrow{\boldsymbol{M}}=\overrightarrow{\boldsymbol{M}}_{o}+\vec{\eta}$ and $\vec{M}, \vec{M}_{o}, \vec{\eta} \in \mathbf{R}^{2}$.

Since $\left.\vec{V}_{\mathbf{V}}, \vec{V}_{2}\right\}$ is a standard basis of $\mathbf{R}^{\mathbf{2}}$, then there exist two standard real numbers $a$ and $b$, and two real infinitesimals $\alpha$ and $\beta$ not both zero, such that

$$
\vec{M}=a \overrightarrow{V_{1}}+b \overrightarrow{V_{2}}+\alpha \overrightarrow{V_{1}}+\beta \overrightarrow{V_{2}}
$$

Now 
if $\alpha \neq \mathbf{0}$, then $\vec{M}=\vec{M}_{o}+\varepsilon_{1} \vec{V}_{1}+\varepsilon_{1} \varepsilon_{2} \vec{V}_{2}$, where $\varepsilon_{1}=\alpha$ and $\varepsilon_{2}=\frac{\beta}{\alpha}$

if $\beta \neq \mathbf{0}$, then $\overrightarrow{\boldsymbol{M}}=\overrightarrow{\boldsymbol{M}}_{o}+\varepsilon_{1} \overrightarrow{\varepsilon_{2} \vec{V}_{1}}+\varepsilon_{2} \overrightarrow{V_{2}}$, where $\varepsilon_{1}=\frac{\alpha}{\beta}$ and $\varepsilon_{2}=\beta$.

Remark:

1- $\quad$ The choice of $a$ and $b$ to be standard and $\alpha, \beta$ to be infinitesimals is necessary, since $\vec{M}_{o}$ is standard and $\vec{\eta}$ is infinitesimal.

2- If $\left\{\vec{V}_{1}, \vec{V}_{2}\right\}$ is a standard usual basis of $\mathbf{R}^{2}$, then

$\vec{M}=(x, y)=\vec{M}_{o}+\varepsilon_{1} \vec{V}_{1}+\varepsilon_{1} \varepsilon_{2} \vec{V}_{2}=\left(x_{o}+\varepsilon_{1}, y_{o}+\varepsilon_{1} \varepsilon_{2}\right)$.

3- If $o\left(x-x_{o}\right)=o\left(y-y_{o}\right)$, then $\vec{M}=(x, y)=\left(x_{o}+\varepsilon, y_{o}+\varepsilon\right)$, where $o$ is denoted to the small oh

4- For any basis $B=\left\{\vec{V}_{1}, \overrightarrow{V_{2}}\right\}$, the matrix form of the decomposition theorem in $\mathbf{R}^{2}$ is given by: $\left(\begin{array}{l}x \\ y\end{array}\right)=\left(\begin{array}{ll}v_{11} & v_{12} \\ v_{21} & v_{22}\end{array}\right)\left(\begin{array}{l}a+\alpha \\ b+\beta\end{array}\right)$, that is $\overrightarrow{\mathrm{X}}=\mathrm{B} \vec{C}$ where $\vec{V}_{1}=\left(\begin{array}{l}v_{11} \\ v_{21}\end{array}\right), \vec{V}_{2}=\left(\begin{array}{l}v_{12} \\ v_{22}\end{array}\right)$ and $\vec{C}$ is the constant matrix $\left(\begin{array}{l}a+\alpha \\ b+\beta\end{array}\right)$, and $B$ is the constant matrix $\left(\begin{array}{ll}v_{11} & v_{12} \\ v_{21} & v_{22}\end{array}\right)$.

\section{Theorem 2.3(General Decomposition Technique Theorem)}

If $\boldsymbol{M}$ is limited point of $\mathbf{R}^{\mathbf{n}}$ whose shadow is ${ }^{\circ} \boldsymbol{M}=\boldsymbol{M}_{\boldsymbol{o}}$, then for $\boldsymbol{M} \neq \boldsymbol{M}_{\boldsymbol{o}}$ there exist $n$ standard vectors $\overrightarrow{\boldsymbol{V}}_{1}, \overrightarrow{\boldsymbol{V}}_{2}, \cdots, \overrightarrow{\boldsymbol{V}}_{n}$ which are linearly independent, and $n$ real infinitesimal numbers $\varepsilon_{1}, \varepsilon_{2}, \cdots, \varepsilon_{n}$ such that $\vec{M} \cong \vec{M}_{o}+\varepsilon_{1} \vec{V}_{1}+\varepsilon_{1} \varepsilon_{2}{\overrightarrow{V_{2}}}_{+}+\cdots+\varepsilon_{1} \varepsilon_{2} \cdots \varepsilon_{n} \overrightarrow{V_{n}}$.

Theorem 2.4(Decomposition Version of Generalized Curvature)

Let $\boldsymbol{M}_{o}$ be a standard point of a standard curve $\gamma$ in $\boldsymbol{C}^{\infty}$, and let $\overrightarrow{\boldsymbol{M}} \cong \overrightarrow{\boldsymbol{M}}_{o}+\varepsilon_{1}{\overrightarrow{V_{1}}}_{1} \varepsilon_{1} \varepsilon_{2}{\overrightarrow{V_{2}}}_{2}$, then the general curvature at $\boldsymbol{M}_{o}$ is independent of the choice of $\boldsymbol{M}$ and it given by $\quad{ }^{o} \kappa_{G}=\left|\frac{\varepsilon_{2}}{\varepsilon_{1}^{\frac{q}{p}-1}}\right|$

Proof:

Let $p$ and $q$ be the respective orders of the first non zero vector derivative $\gamma^{(p)}$ and of the first vector derivative not collinear with $\gamma^{(q)}$.

By using the Taylor development up to the order $q$, and the decomposition theorem, the point $\boldsymbol{M}$ can be written in two different ways: 


$$
\vec{M} \cong \vec{M}_{o}+\varepsilon_{1} \vec{V}_{1}+\varepsilon_{1} \varepsilon_{2} \vec{V}_{2},
$$

and

$$
\vec{M} \cong \gamma\left(t_{o}\right)+\cdots+\frac{\gamma^{(p)}\left(t_{o}\right)}{p !}\left(t-t_{o}\right)^{p}+\cdots+\frac{\gamma^{(q)}\left(t_{o}\right)}{q !}\left(t-t_{o}\right)^{q}+\eta\left(t-t_{o}\right)^{q},
$$

where $\eta \cong \mathbf{0}$ Putting $\boldsymbol{t}-\boldsymbol{t}_{\mathrm{o}}=\delta, \delta \cong \mathbf{0}$ we get

$$
\varepsilon_{1} \overrightarrow{V_{1}}+\varepsilon_{1} \varepsilon_{2} \overrightarrow{V_{2}}=\frac{\gamma^{(p)}\left(t_{o}\right)}{p !} \delta^{p}+\cdots+\frac{\gamma^{(q)}\left(t_{o}\right)}{q !} \delta^{q}+\eta \delta^{q}
$$

Dividing equation (3.4.1) by $\varepsilon_{1}$ we get

$$
\overrightarrow{V_{1}}+\varepsilon_{2} \overrightarrow{V_{2}}=\frac{\gamma^{(p)}\left(t_{o}\right)}{p !} \frac{\delta^{p}}{\varepsilon_{1}}+\cdots+\frac{\gamma^{(q)}\left(t_{o}\right)}{q !} \frac{\delta^{q}}{\varepsilon_{1}}+\eta \frac{\delta^{q}}{\varepsilon_{1}}
$$

Since $\frac{\delta^{p}}{\varepsilon_{1}}$ is limited and $\frac{\delta^{q}}{\varepsilon_{1}} \cong \mathbf{0} \forall \boldsymbol{q}>\boldsymbol{p}$, then from the decomposition theorem, we have

$$
\varepsilon_{1}=\left\|M-M_{o}\right\|
$$

Therefore

$$
\frac{\delta^{p}}{\varepsilon_{1}}=\frac{\delta^{p}}{\left\|\frac{\gamma^{p}}{p !} \delta^{p}+\cdots+\frac{\gamma^{q}}{q !} \delta^{q}+\eta \delta^{q}\right\|}=\frac{1}{\left\|\frac{\gamma^{p}}{p !}+\beta\right\|}, \beta \cong \mathbf{0}
$$

Thus $\frac{\delta^{p}}{\varepsilon_{1}} \cong \frac{p !}{\left\|\gamma^{p}\right\|}$, which is limited. Taking the shadow of the equation (2.4.2) we get $\boldsymbol{V}_{1}=\frac{\gamma^{p}}{\left\|\gamma^{p}\right\|}$ which is a unit vector in $\mathbf{R}^{\mathbf{2}}$. The scalar product of equation (2.4.1) by $V_{1}$ implies

$$
\varepsilon_{1}=\frac{\left\|\gamma^{(p)}\right\|}{p !} \delta^{p}+\cdots+\frac{\gamma^{(q)} \cdot \gamma^{(p)}}{q !\left\|\gamma^{(p)}\right\|} \delta^{q}+\xi \quad, \xi \cong \mathbf{0} .
$$

Then

$$
\varepsilon_{1} \cong \frac{\left\|\gamma^{(p)}\right\|}{p !} \delta^{p}
$$


Again, the norm of the cross product of equation (2.4.1) by $\boldsymbol{V}_{\mathbf{1}}$ is implies

$$
\left|\varepsilon_{1} \varepsilon_{2}\right| \cong \frac{\left\|\gamma^{(q)} \times \gamma^{(p)}\right\|}{q !\left\|\gamma^{(p)}\right\|} \delta^{q}
$$

$\cdots$ (2.4.4)

Now raising equation (2.4.4) to the power $q$ and equation (2.4.3) to the power $p$ and then dividing the obtained two equations we get

$$
\frac{(p !) \frac{q}{p}\left(\gamma^{(q)} \times \gamma^{(p)}\right)}{(q !)\left\|\gamma^{(p)}\right\| \|^{\frac{q}{p}+1}}={ }^{o} \kappa_{G} \cong \frac{\varepsilon_{2}}{\varepsilon_{1}^{\frac{q}{p}-1}}
$$

Corollary 2.5

The curvature $K(t)$ of a standard curve at a standard biregular point is given by

$$
K(t)=2\left|\frac{\varepsilon_{2}}{\varepsilon_{1}}\right| \text {. }
$$

Corollary 2.6

Let $\boldsymbol{M}_{\boldsymbol{o}}$ be a standard point of a standard curve $\gamma$ in $\boldsymbol{C}^{\infty}$, and let $\overrightarrow{\boldsymbol{M}} \cong \overrightarrow{\boldsymbol{M}}_{o}+\varepsilon_{1} \overrightarrow{\boldsymbol{V}}_{1}+\varepsilon_{1} \varepsilon_{2} \overrightarrow{\boldsymbol{V}}_{2}, \quad$ where $\boldsymbol{B}=\left\{\boldsymbol{V}_{1}=\left(\boldsymbol{a}_{1}, \boldsymbol{b}_{1}\right), \boldsymbol{V}_{2}=\left(\boldsymbol{a}_{2}, \boldsymbol{b}_{2}\right)\right\}$ is any standard basis of $\mathbf{R}^{2}$, then we have the following cases:

(1) If $a_{1} \neq \mathbf{0}, b_{1}=0, b_{2} \neq 0$, then ${ }^{o} \kappa_{G}=\left|\frac{\varepsilon_{2} b_{2}}{\varepsilon_{1}^{\frac{q}{p}-1} a_{1}^{\frac{q}{p}}}\right|$.

(2) If $V_{1}$ and $V_{2}$ are basic unit direction vectors, then $\boldsymbol{a}_{i}=\mathbf{1}$, whenever $\boldsymbol{b}_{\boldsymbol{i}}=\mathbf{0}$, and conversely.

(3) For cases other than (1) and (2), $\kappa_{G}$ is unlimited or undefined

Proof: 
(1) Form the following table of all possible values of $\left(a_{1}, b_{1}\right)$ and $\left(\boldsymbol{a}_{2}, \boldsymbol{b}_{2}\right)$ for the basis $\boldsymbol{B}$ we can deduce the result

\begin{tabular}{|c|c|c|c|c|c|}
\hline$a_{1} \neq 0$ & $b_{1} \neq 0$ & $a_{2} \neq 0$ & $b_{2} \neq 0$ & \multicolumn{2}{|c|}{${ }^{o}{ }_{K_{G}}$} \\
\hline Yes & Yes & Yes & Yes & \multirow{3}{*}{$\frac{b_{1} / a_{1}}{\varepsilon_{1} 1^{\frac{q}{p}-1}\left[a_{1}^{2}+b_{1}^{2}\right]^{\frac{1}{2}}}$} & \multirow{3}{*}{$\begin{array}{c}\text { This is either } \\
\text { unlimited or } \\
\text { undefined }\end{array}$} \\
\hline Yes & Yes & Yes & No & & \\
\hline Yes & Yes & No & Yes & & \\
\hline Yes & No & Yes & Yes & \multirow{2}{*}{\multicolumn{2}{|c|}{ 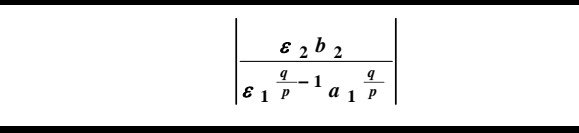 }} \\
\hline Yes & No & No & Yes & & \\
\hline No & Yes & Yes & Yes & \multirow{2}{*}{\multicolumn{2}{|c|}{$\left|\frac{1}{\varepsilon_{1} \varepsilon_{2} a_{2}}\right|$}} \\
\hline No & Yes & Yes & No & & \\
\hline
\end{tabular}

(2) Obviously

(3) In this case, the general curvature is the same as that given by Theorem 2.4, but here we give a simple proof as follows:

Since $V_{1}$ and $V_{2}$ are basic unit direction vectors, then we have $M=(x, y)=\left(x_{o}, y_{o}\right)+\varepsilon_{1}(1,0)+\varepsilon_{1} \varepsilon_{2}(0,1)=\left(x_{o}+\varepsilon_{1}, y_{o}+\varepsilon_{1} \varepsilon_{2}\right)$ Therefore such as shown in the figure (2.1) we get $\left\|M-M_{o}\right\|=\varepsilon_{1}\left(1+\varepsilon_{2}^{2}\right)^{\frac{1}{2}} \cong \varepsilon_{1}$ and $\tan \left(\left.\vec{T}\right|_{M_{o}}\right)=\tan (\theta)=\frac{\varepsilon_{1} \varepsilon_{2}}{\varepsilon_{1}}$ Thus $\left.{ }^{o} \kappa_{G}(\gamma)\right|_{M_{o}}=\left|\frac{\varepsilon_{2}}{\varepsilon_{1}^{\frac{q}{p}-1}}\right|$ 3-Spherical Curvature

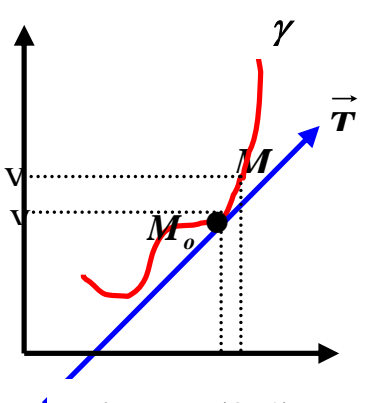

Figure (2.1)

Nonstandard studies of curves are now available widely for geometrical and non geometrical applications, [6], [7], [13].

In this section the problem of curvature on spherical Euclidean space is studied. For planar curves [3], [8], we have only two related order derivatives corresponding to a standard curve, while for spherical curves, three related order of derivatives effect the behavior of the curve, as it is shown in Theorem 3.5.

Other studies on spherical curvatures can be found in [1], [2], [14] and [18]. The characterization of this study begins with the fundamental definition of curvature, which is applicable to any curve in all spaces. 


\section{Theorem 3.1}

Let $\gamma$ be a standard curve of type $C^{\infty}$ in $\mathbf{R}^{3}$ which admits at each point two vector derivatives not coplanar denoted by $\gamma^{(p)}$ and $\gamma^{(q)}$, where $p$ and $q$ are the smallest integers such that $\gamma^{(p)} \times \gamma^{(q)} \neq \mathbf{0}$. Then the generalized curvature of $\gamma$ is given by

$$
{ }^{o} \kappa_{G}(t)=\frac{(p !)^{\frac{q}{p}}\left|\gamma^{(p)} \times \gamma^{(q)}\right|}{q !\left\|\gamma^{(p)}\right\|^{q}{ }^{+1}} .
$$

Proof:

Let $\boldsymbol{B}=\gamma(\boldsymbol{t}+\alpha)$ and $\boldsymbol{C}=\gamma(\boldsymbol{t}+\alpha \varepsilon)$ where $\alpha, \varepsilon \cong \mathbf{0}$ be two tinfinitely close to the point $\boldsymbol{A}=\gamma(\boldsymbol{t})$. By Theorem 1.2, we have

$$
\kappa_{G} \cong \frac{|\tan \hat{A}|}{\|\overrightarrow{B C}\|} \cong \frac{|\overrightarrow{A C} \times \overrightarrow{A B}|}{\|\overrightarrow{B C}\|^{3}}
$$

We consider the following cases concerning the point $\boldsymbol{A}=\gamma(\boldsymbol{t})$

* Case $\mathrm{I} \backslash A$ is a Biregular point:

We have $\quad\|\overrightarrow{B C}\| \cong|(\beta-\alpha)|\left(x^{\prime 2}+y^{\prime 2}+z^{\prime 2}\right)^{\frac{1}{2}}$

Therefore $\|\overrightarrow{B C}\|^{3} \cong\left|(\beta-\alpha)^{3}\right|\left(x^{\prime 2}+y^{\prime 2}+z^{\prime 2}\right)^{\frac{3}{2}}$

Note that we can obtain two different forms according to the given form of the quantity $(\beta-\alpha)^{3}$ as follows:

$$
(\beta-\alpha)^{3}=\left\{\begin{array}{c}
3 \alpha \beta(\alpha-\beta)+\left(\alpha^{3}-\beta^{3}\right) \cong 3 \alpha \beta(\alpha-\beta) \\
2 \alpha \beta(\beta-\alpha)+(\beta-\alpha)\left(\alpha^{2}-\beta^{2}\right) \cong 2 \alpha \beta(\alpha-\beta)
\end{array}\right.
$$

In general, we have $(\beta-\alpha)^{3}=c \cdot \alpha \beta(\alpha-\beta)$, where $c$ is an arbitrary standard constant.

For our purpose we take $(\beta-\alpha)^{3}=\alpha \beta(\alpha-\beta)$, so

$$
\|\overrightarrow{B C}\|^{3} \cong|\alpha \beta(\alpha-\beta)|\left(x^{\prime 2}+y^{\prime 2}+z^{\prime 2}\right)^{\frac{3}{2}}=\mid \alpha \beta(\alpha-\beta)\left\|\gamma^{\prime}(t)\right\|
$$

And

$$
\overrightarrow{A C} \times \overrightarrow{A B}=\left|\begin{array}{ccc}
e_{1} & e_{2} \\
\left(\beta x^{\prime}+\frac{\beta^{2}}{2} x^{\prime \prime}+\delta_{2} \beta^{2}\right) & \left(\beta y^{\prime}+\frac{\beta^{2}}{2} y^{\prime \prime}+\delta_{2} \beta^{2}\right) & \left(z^{\prime}+\frac{\beta^{2}}{2} z^{\prime \prime}+\delta_{2} \beta^{2}\right) \\
\left(\alpha x^{\prime}+\frac{\alpha^{2}}{2} x^{\prime \prime}+\delta_{1} \alpha^{2}\right) & \left(\alpha y^{\prime}+\frac{\alpha^{2}}{2} y^{\prime \prime}+\delta_{1} \alpha^{2}\right) & \left(\alpha z^{\prime}+\frac{\alpha^{2}}{2} z^{\prime \prime}+\delta_{1} \alpha^{2}\right)
\end{array}\right|
$$




$$
\cong \frac{\alpha \beta(\alpha-\beta)}{2}\left(\left(y^{\prime} z^{\prime \prime}-z^{\prime} y^{\prime \prime}\right) e_{1}+\left(z^{\prime} x^{\prime \prime}-x^{\prime} z^{\prime \prime}\right) e_{2}+\left(x^{\prime} y^{\prime \prime}-y^{\prime} x^{\prime \prime}\right) e_{3}\right)
$$

From equation (3.1.2) and(3.1.3) we get the result

* Case II $\backslash A$ is an Only Regular point:

Since $\boldsymbol{A}=\gamma\left(\boldsymbol{t}_{0}\right)$ is an only regular point, then we have $\gamma^{\prime} \neq \mathbf{0}$ and $\gamma^{\prime} \gamma^{\prime \prime}=\gamma^{\prime} \gamma^{\prime \prime \prime}=\cdots=\gamma^{\prime} \gamma^{(p-1)}=\mathbf{0}$. Therefore expanding the curve $\gamma$ using Taylor development up to the order $q$ at points $\boldsymbol{B}=\gamma\left(\boldsymbol{t}_{\boldsymbol{o}}+\alpha\right)$ and $C=\gamma\left(t_{o}+\beta\right)$, we get

$$
\begin{gathered}
\|\overrightarrow{B C}\|^{q+1}=\left|(\beta-\alpha)^{q+1}\left\|\gamma^{\prime}\right\|^{q+1}=\right| \sum_{i=0}^{q+1}\left(\begin{array}{l}
q+1 \\
i
\end{array}(-1)^{i} \beta^{q+1-i} \alpha^{i}\left\|\gamma^{\prime}\right\|^{q+1}\right. \\
\cong(q+1) \alpha \beta\left(\beta^{q-1}+(-1)^{q} \alpha^{q-1}\right)\left\|\gamma^{\prime}\right\|^{q+1}
\end{gathered}
$$

or

$$
\|\overrightarrow{B C}\|^{q+1}=\left|(\beta-\alpha)^{2}(\beta-\alpha)^{q-1}\left\|\left.\gamma^{\prime}\right|^{q+1} \cong \mid \alpha \beta(\beta-\alpha)^{q-1}\right\| \gamma^{\prime} \|^{q+1}\right.
$$

And

$$
\begin{aligned}
& \overrightarrow{\boldsymbol{A C}} \times \overrightarrow{\mathrm{AB}}=\left|\begin{array}{ccc}
e_{1} & \boldsymbol{e}_{2} & \boldsymbol{e}_{3} \\
\sum_{k=1}^{q} \frac{\beta^{k} x^{k}}{k !}+\delta_{2} \beta^{q} & \sum_{k=1}^{q} \frac{\beta^{k} y^{k}}{k !}+\delta_{2} \beta^{q} & \sum_{k=1}^{q} \frac{\beta^{k} z^{k}}{k !}+\delta_{2} \beta^{q} \\
\sum_{k=1}^{q} \frac{\alpha^{k} x^{k}}{k !}+\delta_{1} \alpha^{q} & \sum_{k=1}^{q} \frac{\alpha^{k} y^{k}}{k !}+\delta_{1} \alpha^{q} & \sum_{k=1}^{q} \frac{\alpha^{k} z^{k}}{k !}+\delta_{1} \alpha^{q}
\end{array}\right| \\
& \cong \frac{\alpha \beta\left(\alpha^{q-1}-\beta^{q-1}\right)}{q !}\left(\left(y^{\prime} z^{(q)}-z^{\prime} y^{(q)}\right) e_{1}+\left(z^{\prime} x^{(q)}-x^{\prime} z^{(q)}\right) e_{2}+\left(x^{\prime} y^{(q)}-y^{\prime} x^{(q)}\right) e_{3}\right) \ldots
\end{aligned}
$$

From equation (3.1.5) and(3.1.6) we get

$$
\kappa_{G}(t) \cong \frac{\left(\frac{\beta}{\alpha}\right)^{q-1}-1|| \gamma^{\prime}(t) \times \gamma^{(q)}(t) \mid}{\left|\frac{\beta}{\alpha}-1\right|^{q-1} q ! \|\left.\gamma^{\prime}(t)\right|^{q+1}}
$$

Since $\beta=\alpha \varepsilon$, which implies $\frac{\beta}{\alpha} \cong \mathbf{0}$, then 
Proof:

$$
\kappa_{G}(t) \cong \frac{\left|\gamma^{\prime}(t) \times \gamma^{(q)}(t)\right|}{q !\left\|\gamma^{\prime}(t)\right\|^{q+1}}
$$

Using equations (3.1.4) and (3.1.6) we get

$$
\kappa_{G}(t) \cong \frac{\left|\frac{\alpha \beta\left(\alpha^{q-1}-\beta^{q-1}\right)}{q !}\right|\left|\gamma^{\prime}(t) \times \gamma^{(q)}(t)\right|}{\left|\alpha \beta(q+1)\left(\beta^{q-1}+(-1)^{q} \alpha^{q-1}\right)\right|\left|\gamma^{\prime}(t)\right|^{q+1}}
$$

Since $q$ is an odd number, therefore $\kappa_{G}(t) \cong \frac{\left|\gamma^{\prime}(t) \times \gamma^{(q)}(t)\right|}{(q+1) !\left|\gamma^{\prime}(t)\right|^{q+1}}$.

* Case III $\backslash A$ is a Singular point:

Let $\boldsymbol{A}=\gamma\left(\boldsymbol{t}_{\mathrm{o}}\right)$ be a singular point of order $p-1$ and $q$ the order of the first derivative not coplanar with $\gamma^{(p)}$, then using equation (3.1.5) we get

$$
\begin{aligned}
\|\overrightarrow{B C}\| & =\left(\left(0+\cdots+\frac{\beta^{p}-\alpha^{p}}{p !} x^{p}+i . s\right)^{2}+\left(0+\cdots+\frac{\beta^{p}-\alpha^{p}}{p !} y^{p}+i . s\right)^{2}+\left(0+\cdots+\frac{\beta^{p}-\alpha^{p}}{p !} z^{p}+i . s\right)^{2}\right)^{\frac{1}{2}} \\
& =\left|\frac{\beta^{p}-\alpha^{p}}{p !}\right|\left(x^{(p)^{2}}+y^{(p)^{2}}+z^{(p)^{2}}\right)^{\frac{1}{2}}=\mid \frac{\beta^{p}-\alpha^{p}}{p !}\left\|\gamma^{(p)}(t)\right\|
\end{aligned}
$$

Thus

$$
\|\overrightarrow{B C}\|^{\frac{q}{p}+1}=\|\overrightarrow{B C}\|^{2}\|\overrightarrow{B C}\|^{\frac{q}{p}-1} \cong \mid \frac{\left(\alpha^{p} \beta^{p}\right)\left(\beta^{p}-\alpha^{p}\right)^{\frac{q}{p}-1}}{(p !))^{\frac{q}{p}+1}}\left\|\gamma^{(p)}(t)\right\|^{\frac{q}{p}+1},
$$

$\overrightarrow{A C} \times \overrightarrow{A B} \cong \frac{\alpha^{p} \beta^{p}\left(\alpha^{q-p}-\beta^{q-p}\right)}{p ! q !}\left(\left(y^{(p)} z^{(q)}-z^{(p)} y^{(q)}\right) e_{1}+\left(z^{(p)} x^{(q)}-x^{(p)} z^{(q)}\right) e_{2}+\left(x^{(p)} y^{(q)}-y^{(p)} x^{(q)}\right) e_{3}\right)$, therefore

$$
|\overrightarrow{A C} \times \overrightarrow{A B}| \cong \mid \frac{\alpha^{p} \beta^{p}\left(\alpha^{q-p}-\beta^{q-p}\right)}{p ! q !}\left\|\gamma^{(p)}(t) \times \gamma^{(q)}(t)\right\|
$$

From equations (3.1.7) and (3.1.8) we have; 
$\kappa_{G}(t) \cong \frac{|\overrightarrow{A C} \times \overrightarrow{A B}|}{\|\overrightarrow{B C}\|^{\frac{q}{p}+1}}=\frac{\frac{\alpha^{p} \beta^{p}\left(\alpha^{q-p}-\beta^{q-p}\right)}{p ! q !} \mid\left\|\gamma^{(p)}(t) \times \gamma^{(q)}(t)\right\|}{\left|\frac{\left(\alpha^{p} \beta^{p}\right)\left(\beta^{p}-\alpha^{p}\right)^{\frac{q}{p}-1}}{(p !)^{\frac{q}{p}+1}}\right|\left\|\gamma^{(p)}(t)\right\|^{\frac{q}{p}+1}}$

Since $\quad \beta=\alpha \varepsilon \quad$ which implies $\quad \frac{\beta}{\alpha} \cong 0$ therefore $\kappa_{G}(t) \cong \frac{(p !)^{\frac{q}{p}}\left\|\gamma^{(p)}(t) \times \gamma^{(q)}(t)\right\|}{q !\left\|\gamma^{(p)}(t)\right\|^{\frac{q}{p}+1}}$

\section{Corollary 3.2}

If $\boldsymbol{A}=\gamma\left(\boldsymbol{t}_{o}\right)$ is the biregular point of $\gamma$, then for any value of $\alpha$ and $\beta$, the general case of the generalized curvature of $\gamma$ is given by

$$
\kappa_{G} \cong \frac{\left\|\gamma^{\prime}(t) \times \gamma^{\prime \prime}(t)\right\|}{c \cdot\left\|\gamma^{\prime}(t)\right\|^{3}}
$$

\section{Corollary 3.3}

If $\boldsymbol{A}=\gamma\left(\boldsymbol{t}_{\boldsymbol{o}}\right)$ is the only regular point of $\gamma$ and $q$ is the order of the first derivative which is not collinear with $\gamma^{\prime}$, and $\boldsymbol{B}=\gamma(\boldsymbol{t}+\alpha)$, $C=\gamma(t+\beta)$ are two points infinitely close to the point $A=\gamma\left(t_{o}\right)$, then for any value of $\alpha$ and $\beta$, the generalize curvature of $\gamma$ is given by:

$$
\kappa_{G}(t) \cong \frac{\left|\gamma^{\prime}(t) \times \gamma^{(q)}(t)\right|}{(q+1) ! \|\left.\gamma^{\prime}(t)\right|^{q+1}}, \text { provided that } q \text { is an odd number. }
$$

\section{Corollary3.4}

Let $\boldsymbol{A}=\gamma\left(\boldsymbol{t}_{\boldsymbol{o}}\right)$ be a singular point of order $p-1$ and $q$ the order of the first derivative not coplanar with $\gamma^{(p)}$, and $\boldsymbol{B}=\gamma(\boldsymbol{t}+\alpha), \boldsymbol{C}=\gamma(\boldsymbol{t}+\boldsymbol{\beta})$ be two points infinitely close to the point $\boldsymbol{A}=\gamma\left(\boldsymbol{t}_{o}\right)$. Then for any value of $\alpha$ and $\beta$, the general curvature of $\gamma$ is given by $\kappa_{G}(t) \cong \frac{(p !)^{\frac{q}{p}}\left\|\gamma^{(p)}(t) \times \gamma^{(q)}(t)\right\|}{\left(\frac{q}{p}+1\right) q !\left\|\gamma^{(p)}(t)\right\|^{\frac{q}{p}+1}}$, provided that $\frac{q}{p}$ is an odd number.

Proof:

Since we have 


$$
\begin{aligned}
\|\overrightarrow{B C}\|^{\frac{q}{p}+1}= & \mid\left(\frac{\beta^{p}-\alpha^{p}}{p !}\right)^{\frac{q}{p}+1}\left\|\gamma^{(p)}(t)\right\|^{\frac{q}{p}+1} \\
& =\frac{1}{(p !)^{\frac{q}{p}+1}} \sum_{i=0}^{\left[\frac{q}{p}+1\right]} C_{i}^{\left[\frac{q}{p}+1\right]}(-1)^{i}\left(\beta^{p}\right)^{\frac{q}{p}+1-i}\left(\alpha^{p}\right)^{i}\left\|\gamma^{(p)}\right\|^{\frac{q}{p}+1},
\end{aligned}
$$

thus

$$
\|\overrightarrow{B C}\|^{\frac{q}{p}+1} \cong\left(\frac{\boldsymbol{q}}{p}+1\right) \alpha^{p} \beta^{p}\left(\beta^{q-p}+(-1)^{\frac{q}{p}} \alpha^{q-p}\right) \cdot \frac{1}{(p !)^{\frac{q}{p}+1}}\left\|\gamma^{(p)}\right\|^{\frac{q}{p}+1},
$$

therefore, using equations (3.1.8) and (3.1.9) we get

$\kappa_{G}(t) \cong \frac{(p !)^{\frac{q}{p}}\left\|\gamma^{(p)}(t) \times \gamma^{(q)}(t)\right\|}{\left(\frac{q}{p}+1\right) q !\left\|\gamma^{(p)}(t)\right\|^{\frac{q}{p}+1}}$ provided that $\frac{q}{p}$ is an odd number.

\section{Theorem 3.5}

Let $\gamma$ be a standard curve of type $C^{\infty}$ in $\mathbf{R}^{3}$ that admits at each point three vector derivatives not coplanar, denoted by $\gamma^{(p)}, \gamma^{(q)}$ and $\gamma^{(s)}$, where $p, q$ and $s$ are the smallest integers such that $\gamma^{(p)} \times \gamma^{(q)} \neq \mathbf{0}$, $\gamma^{(p)} \times \gamma^{(s)} \neq \mathbf{0}$ and $\gamma^{(p)} \times \gamma^{(q)} \neq \mathbf{0}$. Then the generalized curvature of $\gamma$ is given by

$$
{ }^{o} \kappa_{G}=\frac{(p !)^{\frac{q}{p}}\left|\gamma^{(p)} \times \gamma^{(q)}\right|}{q ! \mid \gamma^{(p)} \|^{\frac{q}{p}+1}} .
$$

\section{Proof:}

Let $\boldsymbol{B}=\gamma(\boldsymbol{t}+\alpha)$ and $\boldsymbol{C}=\gamma(\boldsymbol{t}+\alpha \boldsymbol{\varepsilon})$ where $\alpha, \boldsymbol{\varepsilon} \cong \mathbf{0}$ be two points infinitely close to the point $\boldsymbol{A}=\gamma(\boldsymbol{t})$. Then expand the curve $\gamma$ using Taylor development up to the order $s$ at each of the points $\boldsymbol{B}=\gamma\left(\boldsymbol{t}_{\boldsymbol{o}}+\alpha\right)$ and $\boldsymbol{C}=\gamma\left(\boldsymbol{t}_{\boldsymbol{o}}+\beta\right)$, we get:

$$
\|\overrightarrow{B C}\|=\left(\left(0+\cdots+\frac{\beta^{p}-\alpha^{p}}{p !} x^{p}+i . s\right)^{2}+\left(0+\cdots+\frac{\beta^{p}-\alpha^{p}}{p !} y^{p}+i . s\right)^{2}+\left(0+\cdots+\frac{\beta^{p}-\alpha^{p}}{p !} \mathbf{z}^{p}+i . s\right)^{2}\right)^{\frac{1}{2}}
$$

thus 
$\|\overrightarrow{B C}\|^{\frac{q}{p}+1}=\|\overrightarrow{B C}\|^{2}\|\overrightarrow{B C}\|^{\frac{q}{p}-1} \cong\left|\frac{\left(\alpha^{p} \beta^{p}\right)\left(\beta^{p}-\alpha^{p}\right)^{\frac{q}{p}-1}}{(p !)^{\frac{q}{p}+1}}\right|\left\|\gamma^{(p)}(t)\right\|^{\frac{q}{p}+1}$

therefore

$$
\begin{aligned}
\overrightarrow{A C} \times \overrightarrow{A B}= & \left|\begin{array}{ccc}
e_{1} & e_{2} & e_{3} \\
\sum_{k=1}^{s} \frac{\beta^{k} x^{k}}{k !}+\delta_{2} \beta^{s} & \sum_{k=1}^{s} \frac{\beta^{k} y^{k}}{k !}+\delta_{2} \beta^{s} & \sum_{k=1}^{s} \frac{\beta^{k} z^{k}}{k !}+\delta_{2} \beta^{s} \\
\sum_{k=1}^{s} \frac{\alpha^{k} x^{k}}{k !}+\delta_{1} \alpha^{s} & \sum_{k=1}^{s} \frac{\alpha^{k} y^{k}}{k !}+\delta_{1} \alpha^{s} & \sum_{k=1}^{s} \frac{\alpha^{k} z^{k}}{k !}+\delta_{1} \alpha^{s}
\end{array}\right|= \\
& \left(\begin{array}{c}
\frac{\alpha^{q} \beta^{q}\left(\alpha^{s-q}-\beta^{s-q}\right)}{s ! q !}\left(y^{q} z^{s}-y^{s} z^{q}\right)
\end{array}\right) e_{1}+ \\
& \left(\begin{array}{c}
\left.\frac{\alpha^{p} \beta^{p}\left(\alpha^{s-p}-\beta^{s-p}\right)}{s ! p !}\left(x^{p} z^{s}-x^{s} z^{s}\right)\right) e_{2}+ \\
\left.\frac{\alpha^{p} \beta^{p}\left(\alpha^{q-p}-\beta^{q-p}\right)}{q ! p !}\left(x^{q} y^{p}-x^{p} z^{q}\right)\right) e_{3}
\end{array}\right.
\end{aligned}
$$

Thus

$$
\begin{aligned}
& \kappa_{G}(t) \cong \frac{|\overrightarrow{A C} \times \overrightarrow{A B}|}{\|\overrightarrow{B C}\|^{\frac{q}{p}+1}}=\left(\frac{(p !)^{\frac{q}{p}+1} \beta^{q-p}\left(\alpha^{s-q}-\beta^{s-q}\right)}{s ! q !\left(\frac{\beta}{\alpha}-1\right)^{\frac{q}{p}-1}\left\|\gamma^{(p)}(t)\right\|^{\frac{q}{p}+1}}\left(y^{q} z^{s}-y^{s} z^{q}\right)\right) e_{1}+ \\
& \left(\frac{(p !)^{\frac{q}{p}} \alpha^{s-p}\left(1-\left(\frac{\beta}{\alpha}\right)^{s-p}\right)}{s !\left(\alpha^{p}\right)^{\frac{q}{p}-1}\left(\left(\frac{\beta}{\alpha}\right)^{p}-1\right)^{\frac{q}{p}-1}\left\|\gamma^{(p)}(t)\right\|^{\frac{q}{p}+1}}\left(x^{p} z^{s}-x^{s} z^{s}\right)\right) e_{2}+ \\
& \left(\frac{(p !)^{\frac{q}{p}}\left(1-\left(\frac{\beta}{\alpha}\right)^{q-p}\right)}{q !\left(\left(\frac{\beta}{\alpha}\right)^{p}-1\right)^{\frac{q}{p}-1}\left\|\gamma^{(p)}(t)\right\|^{\frac{q}{p}+1}}\left(x^{p} y^{q}-x^{q} y^{p}\right)\right) e_{3}
\end{aligned}
$$


Since $\alpha$ and $\beta$ are arbitrary chosen so we may assume that $\frac{\beta}{\alpha}$ is infinitesimal, thus we get

Theorem 3.6

$$
{ }^{o} \kappa_{G}=\frac{(p !)^{\frac{q}{p}}\left|\boldsymbol{x}^{(p)} \boldsymbol{y}^{(q)}-\boldsymbol{x}^{(q)} \boldsymbol{y}^{(p)}\right|}{\boldsymbol{q} ! \mid \gamma^{(p)} \|^{\frac{q}{p}+1}}=\frac{(p !) \frac{q}{p}\left|\gamma^{(p)} \times \gamma^{(q)}\right|}{q !\left\|\gamma^{(p)}\right\|^{\frac{q}{p}+1}}
$$

Let $\gamma$ be a standard curve of type $C^{\infty}$ in $\mathbf{R}^{3}$ which admits at each point two vector derivatives not coplanar denoted by $\gamma^{(p)}$ and $\gamma^{(q)}$, where $p$ and $q$ are the smallest integers such that $\gamma^{(p)} \times \gamma^{(q)} \neq \mathbf{0}$. Then the generalized curvature of $\gamma$ is equal to the shadow $\left|\frac{\varepsilon_{2}}{\varepsilon_{1}^{\frac{q}{p}-1}}\right|$.

Proof:

Form the Decomposition Theorem we have

$\varepsilon_{1} \overrightarrow{V_{1}}+\varepsilon_{1} \varepsilon_{2} \overrightarrow{V_{2}}+\varepsilon_{1} \varepsilon_{2} \overrightarrow{\varepsilon_{3} V_{3}}=\frac{\gamma^{(p)}\left(t_{o}\right)}{p !} \delta^{p}+\cdots+\frac{\gamma^{(q)}\left(t_{o}\right)}{q !} \delta^{q}+\cdots+\frac{\gamma^{(s)}\left(t_{o}\right)}{s !} \delta^{s}+\eta \delta^{s}$

Where $\eta \cong 0$.Dividing equation (3.3.1) by $\varepsilon_{1}$, we get

$\overrightarrow{V_{1}}+\varepsilon_{2} \overrightarrow{V_{2}}+\varepsilon_{2} \overrightarrow{\varepsilon_{3} V_{3}}=\frac{\gamma^{(p)}\left(t_{o}\right)}{p !} \frac{\delta^{p}}{\varepsilon_{1}}+\cdots+\frac{\gamma^{(q)}\left(t_{o}\right)}{q !} \frac{\delta^{q}}{\varepsilon_{1}}+\cdots+\frac{\gamma^{(s)}\left(t_{o}\right)}{s !} \frac{\delta^{s}}{\varepsilon_{1}}+\eta \frac{\delta^{s}}{\varepsilon_{1}}$ thus

$$
\overrightarrow{V_{1}} \cong \frac{\gamma^{(p)}\left(t_{o}\right)}{p !} \frac{\delta^{p}}{\varepsilon_{1}}=\frac{\gamma^{(p)}\left(t_{o}\right)}{p !} \frac{p !}{\left\|\gamma^{p}\right\|}=\frac{\gamma^{(p)}\left(t_{o}\right)}{\left\|\gamma^{p}\right\|}
$$

The cross product of $\boldsymbol{V}_{\mathbf{1}}$ by equation (3.3.1) implies

$$
\varepsilon_{1} \varepsilon_{2} \overrightarrow{V_{1}} \times \overrightarrow{V_{2}}+\varepsilon_{1} \varepsilon_{2} \varepsilon_{3} \times \overrightarrow{V_{1}} \times \overrightarrow{V_{3}} \cong \frac{\gamma^{(p)} \times \gamma^{(q)}}{q !\left\|\gamma^{(p)}\right\|} \delta^{q}+\cdots+\frac{\gamma^{(p)} \times \gamma^{(s)}}{s !\left\|\gamma^{(p)}\right\|} \delta^{s}+\eta \frac{\delta^{s}}{\varepsilon_{1}}
$$

Since each of $V_{1}$ and $V_{2}$ are orthonormal unit vectors then equation (3.3.3) becomes

$$
\varepsilon_{1} \varepsilon_{2} \cong \frac{\gamma^{(p)} \times \gamma^{(q)}}{q !\left\|\gamma^{(p)}\right\|} \delta^{q}, \quad \text { that is } \quad\left|\varepsilon_{1} \varepsilon_{2}\right| \cong \frac{\left\|\gamma^{(p)} \times \gamma^{(q)}\right\|}{q !\left\|\gamma^{(p)}\right\|}\left|\delta^{q}\right|
$$

Moreover from equation (2.4.3) of the proof of Decomposition Version of Generalized Curvature Theorem, we have $\left|\varepsilon_{1}\right| \cong \frac{\left\|\gamma^{(p)}\right\|}{p !}\left|\delta^{p}\right|$ 
Now rising $\left|\varepsilon_{1}\right|$ to the power $q$ and $\left|\varepsilon_{1} \varepsilon_{2}\right|$ to the power $p$, we have

thus

$$
\left|\frac{\varepsilon_{2}}{\varepsilon_{1}^{\frac{q}{p}-1}}\right| \cong \frac{(p !)^{\frac{q}{p}}\left\|\gamma^{(p)} \times \gamma^{(q)}\right\|}{q !\left\|\gamma^{(p)}\right\|^{\frac{q}{p}+1}}={ }^{o} \kappa_{G},
$$

$$
{ }^{o} \kappa_{G}=\left|\frac{\varepsilon_{2}}{\varepsilon_{1} \frac{q}{p}-1}\right|
$$

\section{References}

[1] Antoine, C. G. \& Soriano G.; Weighted curvature approximation- numerical tests for 2D dielectric surfaces, Waves Random Media, Vol. 14, (2004) pp.349-363

[2] Bandos, I. A. \& de Azcárraga, J. A.; Generalized curvature and the equations of $\mathrm{D}=11$, IFIC/04-74, FTUV-05-0101 [arXiv: hep- th/ 0501007 v2] (2005)

[3] Ciarlet, P. G.; An Introduction to Differential Geometry, Lecture Notes Series (2005)

[4] Davis, M.; Applied Nonstandard Analysis, Wiley, New York, (1977).

[5] Diener, F.\& Diener M.; Nonstandard Analysis in Practice, SpringerVerlag, Berlin,HeildeBerg,. (1996).

[6] Fleuriot, J. D.; Theorem Proving in Infinitesimal Geometry, Division of Informatics, University of Edinburgh, $J$ of the IGPL, Vol.9, No3, (2001) pp.447-474

[7] Giordano, P.; Infinitesimal Differential Geometry, Acta Math Univ. Com. Vol. LXXIII, No.2 (2004) pp. 235- 278

[8] Gudmundsson, S.; An Introduction to Riemannian Geometry, Lecture Notes version 1.235 - 9 December (2004)

[9] Keisler, H. J.; Elementary Calculus-2 ${ }^{\text {ed }}$-An Infinitesimal Approach, Creative Commons, 559 Nathan Abbott, Stanford, California, 93405, USA. (2005)

[10] Tahir H. Ismail \& Ibrahim O. Hamad; On Generalized Curvature, To Appear.

[11] Lutz R. and Goze M.; Nonstandard Analysis -A Practical Guide with Application; Lecture Notes in math. (881), Springer, Berlin (1982)

[12] Nelson, E.; Internal set Theory - A New Approach to Nonstandard Analysis, Bull. Amer. Math. Soc, Vol.83, No.6, (1977) pp.1165-1198.

[13] Păsărescu, A.; On Some Applications of Nonstandard Analysis in Geometry, Differential Geometry - Dynamical Systems, Vol. 6 (2004) pp. 23- 30. 
[14] Pendás M. A. \& Luaña V.; Curvature of Interatomic Surfaces I. Fundamentals Journal of Chemical Physics Vol. 119, No. 15,(2003) pp.7633-7643

[15] Robinson, A.; Nonstandard Analysis $2^{\mathrm{ED}}$, North-Holland Pub. Comp.(1974).

[16] Rosinger, E. E; Short Introduction to Nonstandard Analysis, arXiv: math. GM/ 0407178 v1 10 Jul (2004)

[17] Stroyan K. D.; Mathematical Background Foundations of Infinitesimal Calculus-2 ${ }^{\text {ed }}$,Academic Press, Inc (1997).

[18] Velimirovic, L. S. \& Mincic S. M.; Infinitesimal Deformations of Curvature Tensors at Non Symmetric Affine Connection Space; МАТЕМАИЧК ВЕСНИК 54(2002), pp.219-226 